Towards a Decent Labour Market for Low-Waged Migrant Workers 



\title{
Towards a Decent Labour Market for Low-Waged Migrant Workers
}

\author{
Edited by
}

Conny Rijken and Tesseltje de Lange 
Cover illustration: Pulling anchors for post-tensioning cables Source: Wikimedia Commons

Cover design: Coördesign, Leiden

Lay-out: Crius Group, Hulshout

$\begin{array}{ll}\text { ISBN } & 9789462987555 \\ \text { e-ISBN } & 9789048539253 \text { (pdf) } \\ \text { DOI } & 10.5117 / 9789462987555 \\ \text { NUR } & 740\end{array}$

\section{(c) $(1) \Theta \Theta$}

Creative Commons License CC BY NC ND (http://creativecommons.org/licenses/by-nc-nd/3.o)

@ All authors / Amsterdam University Press B.V., Amsterdam 2018

Some rights reserved. Without limiting the rights under copyright reserved above, any part of this book may be reproduced, stored in or introduced into a retrieval system, or transmitted, in any form or by any means (electronic, mechanical, photocopying, recording or otherwise). 